El tránsito de la escuela pública a la escuela privada en el sector emergente de Lima Metropolitana: ¿Buscando mejor calidad?

The transition from public to private school in the rising sector of Lima Metropolitana:

Looking for best quality?

Pilar Sanz

Universidad de Bath mp_sanz@hotmail.com 


\title{
Resumen
}

El tránsito masivo de la escuela pública a la privada en el sector emergente suele ser considerado como una respuesta a la baja calidad de la educación pública. Este estudio sostiene que la opción por lo privado también estaría respondiendo a la formación de una disposición o habitus, que hace que esta opción sea asumida de manera inercial y automática por las familias como concreción de una lógica aspiracional dentro de su trayectoria de movilidad ascendente. A través de la exploración de los procesos de elección de escuela de una muestra de familias, el estudio da cuenta de cómo estos están mediados por la búsqueda de un balance entre diferentes criterios que incluyen costos, condiciones materiales de las escuelas, su localización y cercanía, la búsqueda de espacios seguros para sus hijos o el ratio alumno-profesor, entre otros. Asimismo, las decisiones están fuertemente influidas por estrategias de identificación y diferenciación social en el espacio escolar, en el cual el compromiso con la educación de sus hijos y la composición social de las familias que conforman una escuela resultan factores relevantes tanto para aquellas familias que se identifican entre sí "como uno", como de diferenciación y exclusión de aquellas que representadas como «los otros». De esta manera, se marca una distancia con las familias que envían a sus hijos a la escuela pública o a escuelas de menores recursos. Este estudio busca introducir en el debate un mejor entendimiento del proceso de elección de escuelas, que va más allá de la búsqueda de calidad e incluye procesos sociales más complejos.

Palabras clave: elección escolar, movilidad social, identificación, diferenciación social

\begin{abstract}
The massive transit from public to private school in the emerging sector tends to be considered as a response to the low quality of public education. This study argues that the private option would be also responding to the formation of a disposition or habitus that makes that this option be assumed inertial and automatically by families as a realization of an aspirational logic within their path of upward social mobility. Through the exploration of the processes of school choice in a sample of emerging families the study shows how the decision-making processes are mediated by the search for a balance among different criteria which include costs, material conditions of schools, its location and closeness, the pursuit of getting safe places for their children or the student-teacher ratio, among others. In addition, their decisions are strongly influenced by the strategies of identification and social differentiation in the school space. The parents' strong commitment with the education of their children and the social composition of the families that make up a school are relevant factors for both the identification of those "as one», and the differentiation and exclusion of those who represent «the other», marking a distance from families who send their children to public school or schools of fewer resources. This study seeks to introduce into the debate a better understanding of the process of school choice that goes beyond the search for quality and includes more complex social processes.

Keywords: school choice, social mobility, identification, social differentiation
\end{abstract}




\section{El tránsito de la escuela pública a la escuela privada en el sector emergente de Lima Metropolitana: ¿Buscando mejor calidad?}

\section{Introducción}

Durante los últimos quince años ha venido ocurriendo un fenómeno de crecimiento progresivo de la matrícula privada en educación básica en Lima Metropolitana, que -como señaló Cuenca (2013) en un estudio anterior-, prácticamente, ha duplicado su volumen ${ }^{1}$. En su cartografía de la escuela privada, Cuenca muestra cómo el crecimiento de la matrícula ha tenido variaciones significativas dentro de los diferentes quintiles de pobreza, excepto en el más rico; a partir de ello, la opción por la educación básica privada ha dejado de ser exclusiva de los sectores medios-altos y altos y se ha extendido a los diferentes niveles socioeconómicos. Asimismo, señala que la expansión de la oferta se ha concentrado en los distritos periféricos, particularmente, en la zona norte de Lima con mayor crecimiento económico. Otro aspecto que caracteriza a esta nueva oferta privada es que, lejos de ofrecer una calidad homogénea, estaría reforzando la existencia de circuitos educativos diferenciados, en los que colegios - públicos y privados- ofrecen servicios de diversa calidad al atender poblaciones muy distintas en función de su ubicación residencial y sus recursos económicos (Balarín, 2015; Benavides, León y Etesse, 2014; Cuenca, 2013).

Aunque los datos disponibles para una adecuada caracterización de la oferta privada son todavía mínimos, es posible distinguir, además de la oferta tradicional dirigida a los sectores altos y medio-altos (caracterizada por colegios exclusivos y colegios alternativos de orientación laica o religiosa, y en los que se suele privilegiar el eje académico o el eje vincular), una nueva oferta de circuitos de colegios privados, principalmente, ubicados en las zonas periféricas. Si bien dicha oferta es amplia y diversa, este estudio se enfoca en dos tipos de colegio que han surgido al interior de este grupo: los colegios corporativos y los colegios de bajo costo ${ }^{2}$.

El incremento de la oferta y de la matrícula privada en estas zonas ocurre en un contexto de importante crecimiento económico, que ha contribuido a la expansión de una clase media que dispone de más ingresos y viene invirtiendo

1. Se ha pasado de una matrícula de 515.000 estudiantes en 1998 a una de 949.996 estudiantes en 2014 (Unidad de Estadística Educativa - Escale, Ministerio de Educación, s.f.).

2. Los colegios corporativos funcionan como redes de colegios financiadas por corporaciones interesadas en la educación, que buscan ofrecer una educación de calidad, con infraestructura moderna y a precios asequibles a un sector emergente consolidado con pensiones alrededor de los S/. 650. Por su lado, los colegios de bajo costo ofrecen una educación de baja calidad que opera en condiciones de infratestructura y equipamiento bastante limitadas. Muchos de estos colegios funcionan con pocos estudiantes con un rango de pensión mensual entre los S/. 90 y S/. 250. 
en la educación de sus hijos (Cuenca, 2013; Jaramillo y Zambrano, 2013; Franco, Hopenhayn y León, 2011; Arellano, 2010). Aunque no es propósito del presente artículo entrar al debate en torno a la definición de esta clase media, sí se debe precisar que el estudio se ha realizado desde una perspectiva comparada considerando dos grupos que pertenecen a este sector en expansión. Ambos tienen en común una trayectoria de movilidad ascendente; de ahí, la referencia a ellos como sector emergente ${ }^{3}$. Sin embargo, mientras que uno corresponde a un sector consolidado por el nivel del ingreso familiar, de la educación y ocupación de los padres, el otro representa a un sector más bien precario, que salió de la pobreza pero que aún está en situación de vulnerabilidad, pues podría retornar a ser pobre.

\section{Sobre el estudio de elección escolar}

El interés de este estudio es poder entender el tránsito de la escuela pública a la escuela privada a partir de la comparación de una muestra de familias de ambos sectores sociales, que -a su vez- remiten a los dos tipos de colegio anteriormente especificados. Se puede explicar esta transición mediante el deterioro de la escuela pública, que la descalifica como opción para las aspiraciones de las familias emergentes. Ciertamente, el abandono sistemático que el Estado peruano ha tenido para la provisión de una educación pública de calidad refuerza este argumento y facilita también la creación de un imaginario social en relación con la calidad per se atribuido a lo privado (Cuenca, 2013). Los resultados de la evaluación censal de estudiantes (ECE) corroboran, en general, una diferencia a favor de las escuelas privadas sobre las públicas. Sin embargo, este argumento de búsqueda de una escuela de mejor calidad en lo privado se debilita por la enorme heterogeneidad que caracteriza dicha oferta, debido a la cual no se puede asumir que todas las escuelas privadas, por el simple hecho de ser privadas, sean buenas. Los resultados de las evaluaciones nacionales (ECE) han revelado por tercer año consecutivo que, en los distritos periféricos, los colegios públicos se desempeñaron mejor que los privados (Unidad de Medición de la Calidad Educativa, 2015) .

Entonces, aunque la crisis en la educación pública definitivamente influye en la opción del sector emergente por la educación privada, esta explicación resulta insuficiente y exige que el estudio de este fenómeno sea abordado desde

3. En este estudio, se consideraron como condición de emergencia las modificaciones en las variables de movilidad educacional (años de escolaridad y nivel educativo alcanzado), nivel de ingresos familiares y ocupación del jefe de familia, entre generanciones (abuelos, padres, hijos).

4. Además, estudios como el de Calónico y Ñopo (2007) demuestran que, si bien existen mayores retornos a quienes asistieron a escuelas básicas privadas, los resultados muestran también una amplia dispersión, lo cual quiere decir que el retorno será mayor siempre y cuando los colegios sean de calidad. 
una perspectiva más amplia, que indague -además- cómo los procesos de elección educativa están mediados por procesos sociales más complejos. La hipótesis de este estudio fue que la elección escolar también estaba relacionada con la concreción de la lógica aspiracional, la consolidación y el posicionamiento social de estos sectores emergentes, que han llevado a la conformación de una disposición o habitus hacia la escuela privada que la convierte en la opción natural. El objetivo de la investigación fue conocer mejor los procesos de elección de escuelas privadas en familias de sector emergente en Lima Metropolitana a través de una exploración de dos aspectos: las expectativas y aspiraciones en relación con la educación de sus hijos, y los criterios y disposiciones que han prevalecido en las familias en el proceso de elección de las escuelas privadas. Asimismo, con el fin de explorar cómo se ha formado esta disposición por la educación privada, busqué responder a la pregunta «¿Qué relación existe entre el proceso de elección de escuela privada, y la trayectoria de movilidad ascendente y de consolidación social del sector emergente?».

Se trata de un estudio cualitativo, basado en las narrativas de padres y madres de familia de sectores emergentes sobre la elección de colegio para sus hijos y sobre sus trayectorias educativas familiares; estas han sido recogidas a través de discusiones grupales y entrevistas a profundidad. Se trabajó con una muestra de familias de dos colegios privados ubicados en distintos circuitos educativos: un colegio de consoricio y otro colegio de bajo costo. El primer colegio es uno de los más costosos del distrito de Los Olivos (denominado Colegio A), mientras que el segundo es un colegio de bajo costo, localizado en el distrito de Villa María del Triunfo (Colegio B).

Tabla I. Los colegios del estudio

\begin{tabular}{ll}
\hline \multicolumn{1}{c}{ Colegio de consorcio } & \multicolumn{1}{c}{ Colegio de bajo costo } \\
\hline $\begin{array}{l}\text { Ofrece } \\
\text { inicial, primaria y secundaria. }\end{array}$ & Ofrece \\
Fue creado en el año 2006. & inicial y primaria \\
380 alumnos: & 107 alumnos: \\
30 alumos por aula aprox. & 10 alumnos por aula aprox. \\
\hline
\end{tabular}

Fuente: Elaboración propia

Se empezó realizando las sesiones de discusion grupal, dos sesiones en cada colegio, en las que participó un total de 29 padres y madres de familia. El objetivo de estas sesiones fue conocer los procesos de elección de colegio por parte de las familias, así como las opiniones, posiciones, expectativas y creencias de los padres y madres de familia con respecto a la escuela privada y la escuela pública. En una segunda etapa, se seleccionaron ocho parejas de padres que habían participado previamente de las discusiones grupales y, con ellas, se realizaron las entrevistas en profundidad (en total, cuatro entrevistas en cada colegio). La finalidad de las entrevistas fue explorar la trayectoria educativa 
de las familias y sus trayectorias de movilidad ascendente. En la presentación de los hallazgos, se incluyen diferentes citas de padres y madres que permiten ilustrar mejor sus discursos y prácticas ${ }^{5}$. Antes de la presentación y discusión de los hallazgos, se hará referencia al marco conceptual que orientó el análisis.

\section{La perspectiva racional-estratégica y la perspectiva culturalista en los estudios de elección escolar}

Para comprender los procesos y estrategias familiares que median en la elección escolar y su relación con los procesos de formación y consolidación del sector emergente, se han utilizado dos perspectivas analíticas desarrolladas desde la sociología de la educación, y que están en la base de una larga tradición de estudios sobre elección escolar y mercado educativo (Gewirtz Ball y Bowe, 1995; Hatcher, 1998; Reay y Ball, 1998; Lauder et ál., 1999; Reay, Davies, David y Ball, 2001; Adnett y Davies, 2002; Ball, 2003; Wu, 2011; Kosunen, 2013). Por un lado, está el enfoque racional, basado en la teoría de la acción racional. Este considera a los actores sociales como esencialmente estratégicos, "que actúan de acuerdo a sus intereses buscando máximizar la utilidad de sus decisiones sopesándolas constantemente en función a resultados» (Ball, 2003, p. 17). De acuerdo con Hatcher, «las decisiones de elección educativa se basan en cálculos racionales de costo-beneficio, que consideran tanto los riesgos como las posibilidades de éxito y de fracaso entre diferentes opciones. El éxito se define principalmente en términos de los posteriores retornos económicos» (1998, p. 20). Dentro de esta perspectiva, Goldthorpe señala que la principal preocupación de las familias es que, a través de la trayectoria educativa de sus hijos, ellos «logren obtener las calificaciones y certificaciones (títulos, credenciales) necesarias para poder preservar o mejorar la posición socio-económica alcanzada o, por lo menos, evitar descender a niveles inferiores» (1996, p. 56).

Desde una posición ontológica muy diferente, las explicaciones «culturalistas» de la elección educativa se basan principalmente en el trabajo de Pierre Bourdieu. Según esta perspectiva, las decisiones pueden ser entendidas como la operación de un «sentido práctico» y de una lógica natural, que deriva de una disposición o habitus que deja muy poco a la planificación deliberada o consciente (Ball, 2003). El habitus se define como «la interiorización de ciertas orientaciones, gustos, modos de pensar, actuar, juzgar, percibir y apreciar el mundo» (Bourdieu y Wacquant, 1992, p. 126), que determina «qué cosas hacer o no hacer, qué cosas decir o no decir en relación con un probable futuro próximo» (Bourdieu, 1990, p. 53). Como señala Ball (2003), este es el mundo de lo que dicta el sentido común. De acuerdo con Lauder et ál. (1999), el habitus consituye una especie de «sabiduría de clase», que conduce a que ciertas decisiones se den por sentadas, sean siempre asumidas y funcionen automáticamente,

5. Para proteger el anonimato, los nombres reales han sido reemplazados por nombres ficticios. 
porque es lo que corresponde o se vuelve natural «para nosotros». El uso del concepto de disposición o habitus se complementa con la noción de capital desarrollada por Bourdieu (capital económico, social, cultural y simbólico) y su influencia en los procesos de elección educativa. Como señala Ball, «en la elección de la educación privada puede jugar un rol relevante el capital social y cultural que puede ser acumulado, así como el estatus y prestigio que se atribuye a quienes asisten a un determinado colegio privado (i.e su capital simbólico y de diferenciación)» (1997, p. 2).

Se debe destacar la relación de complementariedad entre las perspectivas racional y culturalista. Como Bourdieu reconoce, hay límites para el alcance explicativo del concepto de habitus: «el habitus es un principio de producción de prácticas entre otros, y aunque sin duda es utilizado con más frecuencia que cualquier otro [...] no se puede descartar que bajo ciertas circunstancias pueda ser reemplazado por el cálculo racional y la deliberación consciente» (Bourdieu, 1990, p. 108). Considerando lo planteado por ambas perspectivas, en la literatura, se distinguen diferentes beneficios que puede generar la educación al ser concebida como un bien y que estarían mediando los procesos de elección escolar. Adnett y Davies (2002) señalan que la educación como bien de consumo se refiere a los beneficios inmediatos que brinda la elección educativa: sensación de bienestar de los hijos en la escuela, satisfacción con el estatus del colegio y su composición social. La educación como bien de inversión alude, más bien, a los beneficios futuros de la elección educativa en términos de oportunidades para continuar con estudios universitarios o poder alcanzar un mejor posicionamiento laboral. Finalmente, la educación como bien posicional se deriva del trabajo de Brown (1990) y su teoría del conflicto de posiciones. Esta noción refiere a los beneficios que da la elección educativa para mantener o mejorar la posición relativa (de las familias) dentro de una determinada jerarquía, que cobra especial interés en sociedades fuertemente segmentadas, como es el caso de la sociedad limeña.

\section{La elección escolar realizada por familias emergentes}

Para la presentación de los hallazgos se plantea, en primer lugar, el valor que tiene la escolaridad para las familias entrevistadas, para lo cual se consideran sus expectativas presentes y futuras, y de qué tipo de escolaridad se está hablando. En segundo lugar, se hace una breve referencia a cómo las familias se involucran en el proceso de elección escolar tomando en cuenta que son nuevos usuarios del sistema privado. La tercera sección se centra en analizar la diversidad de criteros utilizados en su deliberación para elegir la escuela. Finalmente, la cuarta y última sección presenta el análisis del rol que también juegan las disposiciones, y las prácticas de identificación y diferenciación social en el proceso de elección escolar. 


\subsection{El valor de la escolaridad}

La relevancia atribuida a la educación en la trayectoria de este sector emergente ha sido largamente estudiada. Primero, se ha investigado a través de aquello que la antropología peruana denominó «el mito de la educación», del cual la generación de los abuelos fue portadora ${ }^{6}$. Luego, en la generación de los padres, la educación y específicamente la oportunidad de profesionalización representó la piedra de toque para convertirse en clase media (Pedraglio, 2003). En el contexto de la actual generación, surgen diversas preguntas en relación con el valor de la escolaridad: ¿la educación sigue teniendo el mismo valor como medio para viabilizar el futuro del individuo y su familia? ¿La educación contribuye a fortalecer o consolidar su trayectoria de movilidad ascendente? ¿Cómo se relaciona la educación de sus hijos con sus actuales aspiraciones de clase? Si existe esta relación, ¿de qué tipo de educación estamos hablando?

El presente estudio partió de la exploración del valor de la educación en ambos grupos de familias. Para ello, se consideró tanto una perspectiva de futuro como aquello que la educación representa en el presente. Como se señaló en la sección previa, diversos estudios sobre elección escolar distinguen entre la educación como un bien de consumo (referido al presente) y la educación como un bien de inversión (referido al futuro). Ambos permiten un mejor entendimiento de la elección educativa en un contexto de transformación, como el que se presenta para este sector emergente (Reay y Ball, 1997; Reay et ál. 2001; Ball, 2003; Del Cueto, 2004; Wu, 2011; Elacqua, 2012).

Cuando se les pidió a los padres y madres que imaginaran a sus hijos e hijas dentro de quince o veinte años, lo que se encontró fue una expectativa optimista en relación con su futuro. La aspiración común fue que sus hijos se convirtieran en profesionales exitosos. Así, mencionaron una amplia diversidad de carreras y ocupaciones, que incluyen ingeniería, diseño, arquitectura, publicidad, ciencias, veterinaria, política, carreras deportivas o carreras de música y danza. Esta diversidad contrasta con el rango bastante más limitado de carreras por las que optó (o pudo optar) la generación de padres ${ }^{7}$. Asimismo, la mención de la formación universitaria como ruta para poder alcanzar esta aspiración fue indiscutible. Sin embargo, para el sector consolidado, esta trayectoria no termina con la formación universitaria (como sí fue el caso en el sector más precario), sino que continúa con expectativas

6. El mito hace referencia al poder de la educación para la movilización social ascendente. En palabras de Toche, «haber supuesto que el acceso a la educación era una condición casi suficiente para aspirar a un ascenso en la vida» $(2009$, p. 150). Esto se expresa con claridad en este estudio en el mensaje dejado por la generación de los abuelos a los padres y madres entrevistados: «La educación te lleva donde tú quieres».

7. En su mayoría, en el sector consolidado, se han dedicado a ser contadores/as y administradores/as; y, en el sector más precario, a ser técnicos y operarios. 
de estudios de posgrado, experiencias de trabajo o estudios de idiomas en el extranjero.

Además de las expectativas vinculadas con el logro de una profesión, la mayoría de padres y madres también refirieron metas de autorrealización personal, expresadas en términos de que sus hijos e hijas lleven a cabo «lo que realmente les guste hacer, lo que les apasiona, que puedan vivir de eso», o de modo más general «que sean personas felices». En menor medida, se mencionaron cuestiones relacionadas con el carácter emprendedor («que se planteen metas y busquen realizarlas», "que tengan iniciativa y que pongan empeño para alcanzar sus metas») o a características asociadas con la construcción de redes sociales sólidas («que esté rodeado de amigos», «que tenga una familia que la apoye y que ella pueda ayudar»). Esta diversidad de expectativas difiere de la perspectiva minimalista y de corto plazo encontrada en estudios anteriores (Toche, 2009). El optimismo de los padres sobre los logros futuros de sus hijos fue notable, y podría interpretarse como un signo de confianza de la continuidad de su proceso de movilidad ascendente o -por lo menos- de la posibilidad de mantener su estado actual.

Asimismo, les resultaba claro que, en función de la obtención de estos logros, no cualquier tipo de educación constituye «un medio eficaz para acumular credenciales para la realización futura» (Ball, 1997, p. 16). Para ambos grupos, solo la educación privada parece cumplir con esta expectativa. Ello evidencia la lógica de la escolaridad como bien de inversión, en la cual las familias parecen apostar por la «construcción de una trayectoria educativa que permita a sus hijos/as obtener las credenciales suficientes para asegurar un lugar en una buena universidad y así poder alcanzar una profesión» (Reay y Lucey, 2004, p. 36). Los padres expresan con claridad y determinación este propósito: «[...] que sea un profesional, que postule a la universidad como sea, una universidad estatal, la UNI o la San Marcos [...] esa es mi mira desde ahorita, como sea» (madre, Colegio B).

Esto los ha llevado a romper con el sistema educativo estatal del cual han sido usuarios habituales tanto en la generación de los abuelos como en su generación. En contraposición a lo que ocurre en las clases altas y medias altas -en las que la continuidad y el peso de la tradición es la práctica sostenedora del pasado, y se expresa en la elección de las mismas escuelas de generación en generación (Tiramonti y Ziegler, 2008)-, en el caso de estas familias emergentes, se rompe con la tradición familiar de escolaridad estatal. La nueva opción por la educación privada aparece como una estrategia de reconversión para mantener o consolidar la movilidad alcanzada, debido a que la escuela pública no puede ofrecer o garantizar el logro de sus aspiraciones. La educación se asume, entonces, como un bien posicional por los beneficios que dará la elección educativa para mantener o mejorar su posición actual. 


\subsection{Agencia y proactividad como nuevos usuarios del sector educativo privado}

Un primer aspecto a destacar en el proceso de elección escolar es el carácter parcialmente novedoso que representó para los padres la búsqueda y elección de colegio en el sistema educativo privado, considerando que ambos grupos de familias son usuarios nuevos dentro de dicho sistema. Como Ball et ál., señalan, para diferentes contextos, «sus biografías y trayectorias familiares no les han proporcionado experiencias o conocimientos del sistema escolar privado, ni de los contactos sociales y competencias culturales requeridos para orientar de manera efectiva su preferencia por la opción privada» (1996, p. 102). A pesar de ello, ambos grupos de padres han utilizado diversas estrategias para manejar prácticas de búsqueda y selección de escuelas ${ }^{8}$. Esto incluye pedir referencias y recomendaciones de colegios a familiares, amigos y colegas; visitar diferentes centros educativos; asistir a charlas informativas; realizar búsquedas en Internet; pedir citas con promotores y directores de las instituciones educativas; etc. En general, las familias han adoptado una posición activa y empoderada para ejercer su poder de elección, que contrasta con lo que Bourdieu (1986) denomina «docilidad cultural». Esta última se caracteriza por una respuesta reactiva a los acontecimientos, más que por un compromiso proactivo y exigente, como el adoptado por los padres y madres entrevistados. Quizás, lo que mejor ilustra esta actitud se observa en el caso de una madre que decidió buscar una opinión «calificada» para la elección del colegio:

Nosotros [ella y su esposo] le preguntamos a la directora del nido donde iba mi hija qué colegio nos podía recomendar [...]. Cuando supimos del Colegio A, le pedimos a ella que pudiese visitar el colegio para que nos diera su opinión sobre la propuesta, porque ella sabe; nosotros estábamos contentos con el nido y queríamos algo así de bueno para el colegio (Sra. Chura, Colegio A).

De manera similar a lo que muestran otros estudios sobre elección escolar, son las madres quienes ejercen un rol activo y protagónico a lo largo de todo el proceso de búsqueda de información y selección de alternativas, mientras que los padres se suelen incorporar y ejercer un rol determinante para la decisión final (Ansión, Lazarte, Matos, Rodríguez y Vega-Centeno, 1998; Reay y Ball, 1998; Vincent y Ball, 2006).

8. Sin embargo, cabe resaltar que un estudio realizado previamente por Ansión et ál. (1998) refiere al uso de varias de estas estrategias para la elección de escuelas públicas en el contexto urbano en Lima a finales de 1990. 


\subsection{Diversidad de criterios que sopesan las familias al elegir una escuela}

En contraste con lo encontrado en estudios anteriores en relación con las expectativas educativas del sector emergente, caracterizadas por demandas minimalistas e instrumentales (Toche, 2009), lo que los padres entrevistados manifiestan es el uso de una amplia variedad de criterios que son sometidos a evaluación y deliberación en su proceso de toma de decisiones. Estos hacen referencia no únicamente a las condiciones materiales y de infraestructura de los colegios, sino que involucran diferentes consideraciones ${ }^{9}$. Es interesante notar, sin embargo, que los padres de ambos grupos atribuyen diferente importancia a cada uno de los criterios y, por lo tanto, estos influyen de manera diferente en el proceso de evaluación y elección final.

\section{Costo}

Empezamos con este factor, porque el costo de la institución educativa es lo que ha determinado, en primer lugar, su posibilidad de elección. Como señalan Reay et ál., «el costo juega un papel importante en diferenciar lo posible de lo imposible en la elección escolar» (2001, p. 861). Los padres reconocen que la elección de una escuela está fuertemente mediada por el presupuesto familiar y buscan alternativas que sean capaces de pagar: «Aunque no queremos aceptarlo, el colegio privado que elegimos depende de nuestro presupuesto» (padre, Colegio A). En el caso del sector consolidado, los padres entrevistados han podido elegir uno de los colegios privados más caros del distrito (con una mensualidad equivalente a US\$140 mensuales). En cuanto al grupo más precario, los padres han podido elegir dentro del circuito de escuelas de «bajo costo» del distrito una alternativa intermedia, con cuotas equivalentes a US\$ 50 por mes (esta tasa es ligeramente superior a la media dentro de su localidad). Cabe anotar que, si estos costos se comparan con los de los colegios de circuitos privados en distritos más acomodados, se encuentra una brecha considerable ${ }^{10}$. Lo destacable es que, a pesar de que los padres critican esta característica fuertemente segregacionista del sistema educativo, al mismo tiempo, la terminan aceptando y naturalizando ${ }^{11}$ :

9. Esto guarda correspondencia con lo reportado en el estudio de Ansion et ál. (1998) y en las encuestas nacionales de educación -Enaed 2005 y 2007- (Cuenca y Montero, 2006; Montero y Cuenca, 2008).

10. En los distritos de niveles socioeconómicos medio alto y alto, el rango de tarifas de colegios privados va entre los 225 y 400 dólares americanos, y los 400 y 1070 dólares americanos, respectivamente.

11. Ciertamente, esto supone una concepción de «elección», que es bastante restringida y alejada de la noción de agencia, puesto que esté determinada por el poder adquisitivo de las familias. Como señalan Reay y Lucey, en estas condiciones, «la elección escolar enmascara el hecho de ser un marca de privilegio económico» (2003, p. 121). 
Todo lo que es bueno tiene su costo, tiene su precio. Yo creo que el costo de la pensión de este colegio hace que las personas que no tengan los recursos no puedan matricular a sus hijos, muy a su pesar porque saben que el colegio es bueno. Lamentablemente, es así (Sra. Falcón, Colegio A).

\section{Proximidad espacial}

La cercanía de la escuela es otro factor que entra en juego en el proceso de elección. Sin embargo, la importancia atribuida a este criterio difiere entre los dos sectores. Mientras que para el grupo más precario la cercanía de la escuela es un criterio que resulta determinante para la elección, en el sector consolidado, la distancia y localización de la escuela es un factor significativo, que entra en juego y se sopesa junto a otros factores. Como Ball et ál., señalan, en este caso, «hay un trade off entre éstas y otras inquietudes» (1995, p. 62). En cuanto a la ubicación, todas las familias entrevistadas del primer grupo viven cerca de la escuela (llegan caminando y solo en casos excepcionales lo hacen usando transporte público). En contraposición, en el caso del sector consolidado, la composición es más diversa: la mayoría de las familias vive en distritos cercanos a la escuela, un tercio de ellos reside en el mismo distrito y a pocas cuadras de la escuela, y un grupo reducido viene de distritos lejanos.

La elección de escuelas localizadas dentro del mismo entorno barrial responde a poder facilitar cuestiones pragmáticas, de seguridad y control. Como señalaron los padres de familia, esto les permite poder mantener el apoyo de abuelos, y otros familiares para llevar y recoger a sus hijos del colegio, o llevarles el almuerzo. De igual modo, ello les permite evitar desplazamientos largos o complejos, que implican el uso de transporte público y el cruzar avenidas que consideran peligrosas. También, implica poder vigilar o atender rápidamente alguna emergencia que pueda presentarse en el colegio. De acuerdo con lo que plantea Bourdieu (1986), esto corresponde a estilos de vida que se siguen organizando alrededor de un "orden práctico», que busca ajustarse y hacer prevalecer una organización que les ha permitido salir adelante. En el caso del sector consolidado, se encuentra una mayor disposición para llevar a sus hijos a colegios ubicados en zonas más lejanas, lo cual supone sacrificar la organización establecida si esto les permite incorporar nuevos recursos sociales y culturales.

\section{Infraestructura, equipamiento y limpieza}

Los padres también aprecian una buena infraestructura y equipamiento escolar, porque, en general, estos aspectos están muy descuidados en la actual oferta educativa a la que tienen acceso. Como resultado de un mercado desregulado, es muy común encontrar casas "convertidas» en escuelas, que no cumplen condiciones mínimas de infraestructura y mobiliario. Ambos 106 I grupos de padres han elegido escuelas que ofrecen mejores condiciones que el 
promedio de colegios en su zona (aunque la diferencia entre la infraestructura y equipamiento escolar de ambas escuelas es considerable). Cabe destacar que, cuando los padres se refieren a una buena infraestructura, muchas veces, están refiriéndose a condiciones básicas, como se expresa en las siguientes citas:

Aquí [en el colegio] los alumnos tienen carpetas individuales, las aulas tienen una buena iluminación, no están encerrados en un sótano oscuro, los baños están limpios y, lo más importante, la escuela tiene un patio y un patio de colegio. ¡Los estudiantes no tienen que salir a hacer deporte fuera, que es muy peligroso! (madre, Colegio B).

Este colegio nos pareció interesante por la infraestructura. Normalmente, dentro de esta zona, no hay colegios que se encuentren diseñados para ser colegios. Normalmente, es una casa que la convierten en colegio; entonces, tomando en cuenta eso, este colegio era el mejor (Sra. Benites, Colegio A)

En el caso del sector consolidado, la infraestructura de la escuela elegida contrasta notoriamente con la mayoría de las escuelas del distrito y los padres aprecian estas condiciones: «Aquí tienen aulas bonitas, bien iluminadas, buenos baños y ¡hay un parque! [...]; todo eso tienen» (padre, Colegio A). De hecho, poder ofrecer una buena infraestructura es una de las características distintivas del consorcio al que pertenece la escuela. Cabe notar que esto representa y moviliza la lógica aspiracional de los padres, que se enfoca en el colegio como «objeto de deseo», tal como lo expresa esta madre: «Cuando mi esposo vio el impresionante edificio del colegio me dijo: "un día mi hijo estudiará aquí, everigua cuáles son los requisitos de inscripción”» (Colegio A).

\section{Ambientes cálidos y seguros}

Otra preocupación relevante para los padres en el proceso de elección de colegio es poder encontrar un ambiente seguro y cálido para sus hijos. Ambos grupos de padres mencionaron que buscaron escuelas en las que sus hijos pudieran sentirse bien, cómodos y felices. Como Ball et ál. sostienen, «el papel de lo afectivo, del ethos, del ambiente, del sentir, de la impresión, de la sensación y del clima es absolutamente fundamental para la elección educativa» (1996, p. 94). Sin embargo, para ambos grupos, esto encierra matices distintos. Para los padres del grupo consolidado, esto significa que sus hijos reciban en el colegio una atención personalizada en un ambiente cálido y familiar. En ese sentido, aprecian gestos como que los profesores y el personal del colegio reconozcan individualmente a los alumnos y los llamen por sus nombres, o que sean ellos quienes los reciban por las mañanas y así contribuyan a una sensación de familiaridad. Todo ello hace que se sientan cómodos y se justique su «inversión»: «Pagar un colegio privado es un sacrificio para nosotros [padres], pero creo 
que no estoy perdiendo mi dinero cuando veo que mi hijo está aprendiendo, pero sobre todo cuando siento que va feliz haciendo cosas que realmente le gusta» (Sra. Escobal, Colegio A).

Para el grupo de padres del sector más precario, sin embargo, la noción de bienestar de sus hijos en la escuela está relacionada con encontrar un lugar «seguro» para ellos. Esto significa tener profesores que les parezcan confiables para dejarles a sus hijos, tal como lo expresa esta madre: «Puedo irme a trabajar sintiendo que dejo a mi hija en buenas manos» (Colegio B). Este grupo de padres es especialmente sensible a las situaciones de maltrato y abuso infantil que son reportados continuamente por los medios, y -desde su percepciónafectan especialmente, aunque no exclusivamente, a las escuelas públicas. Por esta razón, las relaciones amicales o familiares con los promotores/as o directores/as de las escuelas juegan un rol fundamental para lograr esta sensación de confianza, que los lleva a terminar de elegir o descartar definitivamente un colegio. Esta preocupación por la felicidad de los hijos en los estudios sobre elección escolar es generalmente atribuida a la predominancia de la gratificación en el corto plazo sobre la gratificación diferida que es propia de las clases medias (Ball et ál., 1995). Sin embargo, en este caso, la preocupación por el bienestar y la felicidad que los hijos pueden experimentar en el colegio podría estar reflejando una tendencia nueva para el sector emergente: poder sentirse «como en casa» en la escuela, una vivencia que las generaciones anteriores no lograron experimentar.

\section{Ratio de alumnos por clase}

Otro criterio que ambos grupos de padres valoran son ratios reducidos de alumnos por profesor, aunque dichos ratios varían entre los dos grupos. Los padres del Colegio A consideran como aceptable aulas de hasta 25 o 30 estudiantes ( «nos ofrecieron que en los salones no iban a ser más de 30 alumnos, no 40 o 45 como en los colegios estatales donde no es posible una atención personalizada», padre Colegio A). En cambio, los padres del Colegio B, apelando al mismo criterio de atención individualizada, consideran ratios más reducidos, de quince o dieciséis estudiantes:

Es como con las familias cuando tienes muchos hijos, ¿no? No le puedes dar mucha atención a cada uno. Cuando hay pocos alumnus, yo creo que el profesor conoce a cada uno de ellos, evalúa a cada uno. Manejar pocos alumnos en clase es una ventaja (padre, Colegio B).

Sin embargo, también reconocen que un tamaño pequeño de clase no es una condición suficiente para una buena enseñanza, pues hay escuelas que, a pesar de tener pocos alumnos por clase, no son buenas (en este caso, hacen referencia principalmente a colegios particulares de bajo costo). Finalmente, 108 I en algunos casos, los padres también señalan que, debido a que sus hijos tienen 
ciertas características particulares (son muy inquietos o se distraen fácilmente), les conviene estar clases con pocos alumnos.

\section{Métodos de enseñanza}

Ambos grupos de padres expresaron entusiasmo sobre el uso de metodologías más activas para el aprendizaje en las escuelas elegidas, en comparación con los métodos «aburridos y pasivos» que, según su propia experiencia, se sufre en la escuela pública. También, se manifestaron sensibles y a favor de la idea de respetar los ritmos de aprendizaje de los estudiantes. Asimismo, indicaron que el proceso educativo se debe centrar en los niños, un aspecto que no es considerado en algunas propuestas que prevalecen en el mercado educativo: «A mí me gustó que, en el colegio, dijeran que no era imprescindible que desde pequeños [desde jardín] aprendan a leer o aprendan las matemáticas, que no hay que saturarlos, sino motivarlos más, enseñarles de manera didáctica» (Sra. Chura, Colegio A). En esa línea, varias de las madres entrevistadas, especialmente del Colegio A, rechazaron enérgicamente las propuestas que provienen, según ellas, de colegios de tipo "preuniversitario», en los que los niños son sometidos a una fuerte presión en términos de una alta carga de tareas, acumulación de información, horarios extendidos, exámenes continuos, y aprendizaje de materias y habilidades que corresponden a grados más avanzados:

Una vecina me dijo [en relación con el Colegio A] «dicen que en ese colegio los estudiantes no avanzan». Le respondí: «Ok; si quieres que tu hijita haga tareas y exámenes todo el día, y que esté toda ansiosa, entonces haces bien; no la pongas en "mi colegio". Allí, los niños aprenden jugando, se divierten y aprenden cosas de acuerdo a su edad. Los profesores no esperan que los niños en primer grado aprendan temas que corresponden al segundo o tercer grado [...]» (madre, Colegio A).

En algunos colegios, les exigen entrar desde las 7:15 am y se quedan hasta las 4 de la tarde! Eso es un abuso con los niños. Si me pongo en el lugar de mis hijos, eso no me hubiera gustado (madre, Colegio B).

Madres y padres cuestionan el modelo de aprendizaje que se centra principalmente en acumular información y se preguntan si esto conduce a los niños a aprender lo que realmente necesitan:

Estamos en un mundo en el que la información está cada vez más a la mano; si algo no te enseñaron en el colegio, googleas y sacas información. El colegio debe enseñar habilidades para investigar, así pueden buscar información en la red o en cualquier otro espacio (Sr. Llerena, Colegio A). 


\section{Desarrollo de habilidades personales y sociales}

Otro aspecto que los padres aprecian, especialmente los que pertenecen al sector consolidado, es que la escuela promueva el desarrollo de habilidades personales y sociales. Esto responde al valor que este tiene para la formación integral de sus hijos, puesto que consideran que los prepara para las exigencias que tendrán en la universidad, y porque constituyen una ventaja comparativa en el mercado laboral en el futuro. En cuanto a las habilidades personales, mencionan el liderazgo, la autonomía, la confianza y la desenvoltura. En relación con ello, aprecian las intervenciones de los profesores, quienes se preocupan por que sus hijos logren desarrollarlas:

La profesora de mi hija me dijo un día que quería hablar conmigo y me dijo «Señora Miranda, su hija es muy tranquila; tiene que ser más desenvuelta». Al principio, me sorprendió, porque en el colegio anterior que estuvo mi hija siempre me felicitaban por su buen comportamiento. Pero, luego, me di cuenta de que sí pues, mi hija era demasiado obediente [risas]. Lo que ha logrado acá es eso: se ha desenvuelto; ya no solo obedece sin decir nada [...]. Aquí ha aprendido a ser más suelta y decir qué piensa (Sra. Miranda, Colegio A).

Con respecto a las habilidades sociales, los aspectos más apreciados por este grupo de padres fueron el desarrollo de capacidades de trabajo en equipo y el que puedan desenvolverse en exposiciones orales, pues consideran que «el método del colegio los está preparando para la universidad, porque cuando vayan allá no van a tener ese temor de enfrentarse a un gran público, van a sentirse más sueltos [...]. Eso yo nunca tuve en el colegio» (Sra. Falcón, Colegio A).

\section{Posibilidad de control y exigibilidad de parte de los padres}

Finalmente, en el discurso de los padres, el hecho de pagar por la educación de sus hijos está asociado a que esto les permite influir en el servicio ofrecido. En su opinion, la relación contractual les da el derecho y la posiblidad de intervenir de manera eficaz para solicitar cambios y mejoras, y poder evitar malas prácticas, como el abuso o maltrato infantil. Esto difiere de lo que sienten que sucede en la escuela pública, donde consideran que no hay alguien realmente responsable ni alguien que tenga la capacidad para tomar decisiones eficaces, por lo que quejarse es una pérdida de tiempo:

[...] el colegio es como una empresa; por lo tanto, lo que me está ofreciendo es un servicio y que lo que nos cobra es por ese servicio que nos da, y en esa relación yo puedo exigir. Si yo acepto esa tarifa, puedo exigir que me dé un producto de calidad, que me dé de acuerdo a las expectativas, que cumpla con lo que ofrece y justamente que se desarrollen 
como yo lo deseo [...]. Aquí podemos cambiar alguna de las variables; en cambio, en el Estado, debo aceptar nomás lo que me da [...]. (Sr. Escobal, Colegio A).

En los colegios nacionales, los profesores, porque están nombrados, se sienten seguros en el trabajo y ya no se quieren capacitor, no se dedican. ¿ $\mathrm{Si}$ a nuestros hijos les toca una profesora así? ¡Va a perjudicar su educación! Siempre existe ese temor y no podemos hacer nada (padre, Colegio B).

Es interesante notar que el discurso de los padres muestra, también, cómo las familias están aprendiendo, y haciendo uso de estrategias y prácticas que caracterizan a las dinámicas del mercado, como el uso de formas alternativas para responder a la prestación de servicios propuesto por Hirschman (1970): salida, voz y fidelidad. Precisamente, una de las referencias de los padres -citadas previamente- da cuenta de cómo el tener que pagar por un servicio les da el derecho a reclamar por su calidad (derecho a ejercer voz) o a dejar las escuelas cuando consideran que dan un mal servicio (salida). En relación con esto último, algunos padres afimaron que, si bien estaban dispuestos a aceptar que -como en cualquier negocio- los dueños o promotores del colegio obtengan un beneficio, no están dispuestos a que este sea maximizado a costa de no invertir en la mejora de la calidad del servicio educativo. Este aspecto ha llevado a varias familias a la decisión de cambiar a sus hijos de colegio a pesar de la complejidad de esta decisión:

Al principio, durante el preescolar, estábamos contentos con la escuela. Pero, cuando nuestro hijo empezó la primaria, aunque la escuela decía seguir con el método Montessori y el desarrollo de las inteligencias múltiples, nos dimos cuenta que esto era solo «marketing» y que no lo estaban cumpliendo, así que decidimos cambiarlo de escuela (Sra. Llerena, Colegio A).

Eso nos pasó en el otro colegio. Nosotros estábamos pagando, pero nos dimos cuenta que se estaban yendo los buenos maestros. ¿Qué pasaba? Los dueños del colegio ya se estaban dedicando solo a cobrar. Entonces, fue una decisión drástica, pero buscamos otro colegio y los cambiamos (Sra. Quispe, Colegio A).

Como se plantea desde la perspectiva racionalista, la diversidad de criterios descritos y analizados en esta sección son considerados por los padres y madres entrevistados para el proceso de elección de escuela. Estos son sopesados y sometidos a cálculos de costo-beneficio que toman en cuenta tanto el beneficio inmediato -la sensación de bienestar de los hijos en la escuela- como el beneficio futuro, es decir, su aporte a la trayectoria educativa que buscan 
asegurar para ellos. En este caso, esto se relaciona con la posibilidad de continuar con estudios universitarios para lograr su profesionalización.

\subsection{El lugar de las disposiciones y habitus en la elección escolar}

Como afirma Ball, «el uso de la lógica y del sentir se entrelazan en la elección escolar» (2003, p. 59). Junto con los diferentes criterios indicados en la sección anterior, los padres también expresan cómo fuertes dimensiones emocionales se involucran en el proceso de elección, que son influidos -a su vez- por la interacción de valores y principios. Estas dimensiones se refieren a características de identificación y de diferenciación social, de esfuerzos por establecer los límites del «nosotros», y de estrategias de cierre social ${ }^{12}$ para separarse de «los otros», los cuales están interiorizados como disposiciones o habitus (Bourdieu, 1986) que hacen que ciertas decisiones se den por sentadas y se asuman naturalmente como lo establece el sentido común ${ }^{13}$. En ello, infuye la trayectoria de movilidad ascendente de estos grupos, que posiciona a la escuela como un bien posicional e instrumento de consolidación. Esta sección examina aspectos significativos del discurso de los padres, y analiza cómo están influidos por su proceso de ascenso y consolidación social. Al igual que en la sección anterior, las similitudes y diferencias encontradas entre las dos fracciones estudiadas son mencionadas y discutidas.

Las prácticas de identificación social: Un colegio para «gente como nosotros»

En la búsqueda y elección de escuelas, un aspecto relevante lo constituye el poder encontrar en ellas personas «como uno», lo cual fue mencionado por padres de ambas fracciones y es expresado claramente por esta madre cuando afirma: «Donde tú has escogido el colegio va más o menos con las familias similares a la tuya» (Sra. Benites, Colegio A). Como señala Ball, «no es solo la escuela y su personal los que están sujetos a escrutinio, sino también las familias que pertenecen a ella» (2003, p. 59). En este proceso, los padres mencionan tanto elementos de identificación como de diferenciación. Por un lado, está el reconocimiento de los demás que son "como uno», una especie de «juicio atributivo de clase» (Bourdieu, 1986, p. 473), y, por otro lado, el sentido de ser diferente de los «otros», aquellos que no son "como uno», y que representan aquellos grupos sociales de los cuales desean separarse y diferenciarse.

Se empezará analizando la prácticas de identificación, en las que la fracción emergente más consolidada reconoce una mayor variedad de características

12. Como explica Parkin, el cierre social se refiere a «la práctica de conservación de privilegios al restringir el acceso de otras personas a los recursos y recompensas atribuidos a un grupo social determinado» $(1979$, p. 44$)$.

112 13. El proceso de formación de estas disposiciones o habitus corresponde a otra parte del estudio que no se incluye en este artículo. 
atributivas de clase a través de las cuales se busca afianzar las relaciones con «los semejantes» (Del Cueto, 2004). Los padres mencionaron principalmente dos prácticas o características distintivas de las familias que pertenecen a los colegios que han elegido. La primera práctica es compartida por ambos grupos y se refiere a reconocerse como padres que apuestan y participan activamente en la educación de sus hijos. La segunda se refiere al carácter emprendedor que caracteriza a las familias, lo cual es referido principalmente y de manera casi exclusiva por las familias del sector consolidado.

\section{- Padres preocupados y comprometidos con la educación de sus hijos}

Aunque esta preocupación y compromiso se empieza a manifiestar en el hecho de pagar por su educación, va más allá. Como señala uno de los padres, «no se trata solamente de pagar y que el colegio se encargue, sino estar atentos a lo que sucede en el colegio». Esto significa para ellos ser padres: involucrarse en la vida escolar y monitorear el progreso de sus hijos en el colegio. Para muchos padres, este tipo de compromiso es una característica que los distingue de otras familias que dejan que sus hijos salgan adelante solos, sin ocuparse de ellos ni apoyarlos. Esta preocupación compartida los lleva a reconocer un sentido de «nosotros»: «Los que mandan a sus hijos a este colegio tienen mayormente el mismo objetivo: todos quieren invertir en sus hijos. Yo lo he notado [...] y porque estamos preocupados por nuestros hijos nos metemos en su educación» (madre, Colegio A).

«Los padres que están aquí tienen mucho en común, comparten los mismos valores que nosotros tenemos; es decir, historias que desde antes tenían mucho en común con nosotros, queremos que nuestros hijos sean mejores, no solo que aprendan valores sino que los practiquen. En otros [padres], yo encontré conformismo; piensan que tienen que dejar solos a sus hijos, que encuentren su futuro por ellos mismos» (padre, Colegio A).

Como afirma Ball, todas estas inquietudes y expectativas comunes les dan a los padres un sentido de pertenencia a este lugar que perciben como «su lugar» (2003, p. 59). Por el contrario, para ambos grupos de padres, los «otros» están representados por aquellos que son despreocupados con respecto a la educación de sus hijos, quienes se asocian principalmente a los que envían a sus hijos a las escuelas públicas: «He notado que los padres cuyos hijos están en las escuelas públicas no dedican tiempo a sus hijos, no pasan tiempo con ellos. Muchos tienen que trabajar todo el tiempo y descuidan a sus hijos» (madre, Colegio B). Sin embargo, esta falta de preocupación también es percibida en las escuelas de sus hijos, pero como casos excepcionales: «Acá también hay papás que lo que más les preocupa son sus negocios o su carrera, y solo depositan a sus hijos en un buen colegio, pero nunca se les ve por aquí» (padre, Colegio 
A). Estas respuestas expresan modos de distinción y clasificación, «que sirven para reproducir oposiciones y asociaciones entre escuelas y clases o grupos sociales» (Ball et ál., 1996, p. 95).

En el discurso de los padres, diversas razones explican esta disposición a un compromiso y participación activa en la educación de sus hijos. Antes de analizarlas, cabe destacar que esta participación involucra principalmente a las madres e implica acompañar la rutina escolar, hablar regularmente con el profesor o profesora de la clase, ayudar con las tareas escolares, hablar y coordinar con otros padres, llevar a los hijos a otras actividades extraescolares ${ }^{14}$. En el caso de las familias entrevistadas, la mayoría de madres brindan una dedicación casi exclusiva a la crianza de los hijos. En menor medida, dicha dedicación es compartida con trabajos ocasionales o a tiempo parcial; excepcionalmente, comparten la crianza con trabajos a tiempo completo. Parecería que para ambas facciones de familias emergentes el "paquete» de inversión en educación privada para sus niños se complementa, y potencia con la dedicación de las madres a la crianza y al seguimiento de la escolaridad de sus hijos.

Una primera razón que explica este fuerte involucramiento de las madres está relacionada con experiencias que ellas vivieron y que no quieren repetir con sus hijos. Los padres de ambos grupos refieren que tuvieron un patrón de crianza en el que no se beneficiaron de la presencia de sus padres, porque ellos estaban totalmente dedicados a sacar adelante sus negocios, su trabajo y sus familias (por entonces, bastante numerosas). Aunque valoran el esfuerzo realizado por sus padres para tratar de asegurar un mejor futuro para ellos, reconocen que en su trayectoria su situación ha cambiado y buscan dar a sus hijos lo que ellos no tuvieron: en este caso, la presencia cercana de las madres:

Él [en referencia a su esposo], porque su mamá trabajaba, yo veo que como que no quiere que a los chicos les falte su mamá. A mí no me faltó mi mamá; si bien es cierto no me ha ayudado en las tareas, yo sabía que mi mamá estaba en casa. Si a mí me pasaba algo, yo iba a mi casa y sabía que iba a haber una persona adulta que me ayudase [...]. Por eso, ahora que he terminado mi carrera y puedo trabajar, para mí, es clarísimo que en las tardes no voy a trabajar. Si trabajo, va a ser en las mañanas, mientras ellos (sus dos hijos) estén en el colegio. Si no encuentro un trabajo que me permita eso, entonces, no [...]. Eso es lo que tenemos claro, ¿no? (Sra. Soto, Colegio B).

Asimismo, los padres entrevistados señalaron que la presencia cercana de las madres en la educación y en otras actividades que realizan los hijos asegura un entorno más seguro para ellos, pues los entornos y condiciones en los que viven los consideran algo inseguros. También, reconocen que la presencia

14. Este involucramiento casi exclusivo de las madres es un patrón reportado por diferentes estudios (Reay, 1997; Ansion et ál., 1998; Reay y Lucey, 2003). 
más activa de las madres refuerza o permite potenciar los aprendizajes en la escuela y hacen referencia a la influencia del capital cultural de las familias en los aprendizajes. Algunas madres explican esto poniendo como contraejemplo situaciones que, según ellas, ocurren en los colegios públicos:

En los colegios públicos, he notado que los papás no le dedican tiempo a sus hijos, no les ayudan con sus tareas; $y$, entonces, el nivel académico no va a poder subir, porque cuando los niños están descuidados la profesora no puede avanzar (madre, Colegio B).

\section{- Colegios para gente emprendedora}

Otra característica atributiva con la se identifica el grupo de padres y madres entrevistados fue mencionada a menudo y casi exclusivamente por el grupo consolidado, y se refiere a la correspondencia de la escuela con el espíritu emprendedor que caracteriza a personas "como ellos». En ese sentido, los padres valoran el hecho de que la escuela elegida está dirigida a personas emprendedoras, como lo expresa la Sra. Llerena:

Es un colegio para gente emprendedora, un tipo de educación que no sea demasiado cara, a la que se pueda tener acceso, que tengamos beneficios y que nos ayuden a avanzar a este grupo de personas que somos de los conos [...]. Lo que pasa es que el emprendedor, para que no haga las cosas equivocadamente, tiene que tener he-rramientas; si no está bien educado, se va a equivocar y va a tener mucho estrés, pero, si es una persona educada y tiene ese espíritu de avanzar, entonces se potencia, ¿no? (Colegio A).

En el discurso de los padres y madres, este reconocimiento del espíritu emprendedor como una marca de identidad positiva de su grupo social les hace merecer un tipo particular de escuela, que corresponde a su nuevo estatus y que es muy diferente a la escuela pública que ellos experimentaron. Además, esperan que la experiencia de escolaridad de sus hijos les permita desarrollar o fortalecer actitudes y habilidades de los emprendedores. En ese sentido, buscan un alineamiento entre el habitus de la familia o del grupo social con el habitus de la escuela:

En la escuela, los niños necesitan un entorno que les permita desarrollar algunos elementos básicos que pueden ver en sus padres, como el esfuerzo, el compromiso, la responsabilidad. Estas son cosas que no son fáciles de enseñar, ¿no? Eso se aprende vivencialmente (Sr. Miranda, Colegio A). 
Como indica Ball, «es en la configuración de estos espacios donde el habitus de la familia y el de la escuela se complementan» (2003, p. 59). Además, en este tipo de complementariedad, los padres consideran que la escuela constituye un espacio donde los niños pueden adquirir redes especializadas del conocimiento, de forma que, junto con el desarrollo de un espíritu emprendedor, se construye una atractiva «ecuación» que facilitará la consolidación de sus trayectorias.

Es interesante notar que este espíritu emprendedor, además de representar una característica distintiva de este sector social, refleja también la conciencia de su valor social. Como Reay y Ball (1997) sugieren, este sentimiento colectivo sobre su propia valía social influye en su proceso de elección escolar. Por ello, para el grupo de padres entrevistados, a pesar de su pobre experiencia de escolarización y de ser usuarios novatos del sistema privado, esto no los ha llevado a patrones de elección educativa caracterizados por «la ambivalencia y la búsqueda para evitar la ansiedad, el fracaso y el rechazo» (Ball et ál., 1996, p. 102). Por el contrario, los padres actúan muy activos, claros y decididos para encontrar la «mejor» escuela para sus hijos, la escuela que se merecen como personas emprendedoras, así como un tipo de educación que reconozca y refuerce su identidad colectiva.

En relación con este último aspecto, una diferencia importante entre el discurso de los padres de ambas fracciones es que, mientras que en el grupo consolidado referían buscar «el mejor» colegio para sus hijos (dentro de sus posibilidades económicas), los padres del grupo más precario lo expresaba de manera más modesta. En su discurso, señalaban que lo que buscan es básicamente ofrecer una mejor alternativa a aquella que ofrece la educación pública, como lo indica este padre del Colegio B: «Cuando tú retiras a tu hijo de un colegio estatal y lo llevas a uno privado, esperas un beneficio adicional, esperas algo mejor». Un contraste entre ambos tipos de discurso se muestra en estas dos afirmaciones:

[...] Los niños ya vienen formados desde la casa y el colegio les da un acabado. Entonces, hay que buscar al mejor artesano, porque es la mejor joya lo que estamos dando; y eso es lo que yo busco del colegio (padre, Colegio A).

Mi hija fue primero a una escuela privada y, luego, le cambié a una estatal y vi la diferencia. Hubo una disminución al $20 \%$ en su proceso de aprendizaje en la escuela estatal. Entonces, decidí que mi hija tenía derecho a recibir algo mejor en una escuela privada, donde ella pudiera tener realmente una oportunidad (madre, Colegio B).

\section{Prácticas de diferenciación social: ¿A mezclarse?}

Como ya se señaló, el sentido de identificación social analizado en la sección 116I anterior supone también un sentido de diferenciación. En esta sección, se 
examinan diferentes estrategias identificadas en los discursos y las prácticas de las familias que hablan de los esfuerzos por separarse y distinguirse de «los otros», representados por aquellos grupos con los que no desean ser asociados. Aunque esto puede adoptar muchas formas, aquí el análisis se enfoca en la práctica de búsqueda de una «adecuada» mezcla o composición social en la escuela, en la estigmatización de la escuela pública, y en la búsqueda de estatus y exclusividad que otorga la elección de un determinado tipo de escuela.

\section{- La importancia de la composición social y la estigmatización de la escuela pública}

Una muestra de la dinámica de identidad y diferenciación con la que las familias construyen claramente los bordes sociales se expresa en el nivel de heterogeneidad o mezcla social esperada dentro de la escuela. Durante las discusiones, se evindenciaron diversos puntos de vista con respecto a las expectativas de los padres y madres sobre la composición social deseable para la escuela de sus hijos; este fue un tema más relevante y sensible dentro del grupo más consolidado.

Para la mayoría de los padres entrevistados, la indagación por la composición social de las familias dentro de la escuela dio lugar a ciertos conflictos y contradicciones, especialmente en relación con los principios de igualdad y no discriminación. Por un lado, los padres consideraban positivo que la escuela pudiera reflejar la heterogeneidad que caracteriza al país como parte de la preparación para la vida en una sociedad diversa; de esta manera, rechazaron la idea de discriminación. En esta posición, el propósito instrumental de la mezcla social para que sus hijos aprendan a manejarse frente a lo problemático que puede resultar la convivencia con otros diferentes resulta claro:

Así como la universidad suele ser un Perú pequeñito que te enfrentas con todas las cosas que trae la sociedad, el colegio también suele ser una sociedad en pequeño, ¿no? Ahí ellos [los hijos] aprenden ciertos elementos de capacidad para enfrentar los problemas difíciles (Sr. Llerena, Colegio A).

Sin embargo, lo que no resulta tan evidente en el discurso de los padres es que dicha integración pretenda promover «una sana convivencia entre estudiantes de diferentes procedencias, que en última instancia conduzca a una ciudadanía más tolerante y de mente abierta» (Gill, 2005, p. 131). Solo algunos padres, de manera casi excepcional, destacan esta última perspectiva haciendo referencia precisamente a los colegios estatales:

En el colegio estatal, los niños saben defenderse; como tienen compañeros de diferente carácter, de diferente estatus social, de familias con diferentes problemáticas ahí los niños aprenden a defenderse, pero también 
a tener sensibilidad con sus compañeritos, aprender a ser más sociables entre ellos (Sra. Soto, Colegio B).

Cuando se indagó sobre qué consideraban ellos y ellas que sería un «buen balance» o una «buena mezcla», los padres se sentían más cómodos con la idea de que la heterogeneidad constituyera la excepcionalidad y no la regla. Es decir, la presencia de heterogeneidad en pequeñas cantidades resultaba aceptable ${ }^{15}$. En casos extremos, algunas madres expresaron clara y abiertamente su preferencia por no mezclar, especialmente con niños que provienen de familias en condiciones de riesgo:

Yo no quiero mezclar. Sé que suena feo [baja el tono de voz], pero yo no quiero que mi hijo se mezcle con otros niños que no han sido criados con los principios que yo quiero para mi hijo. ¡Así que no voy a enviarlo para que estudie con el hijo de un ratero o de una drogadicto! (Sra. Chura, Colegio A).

En este punto de la discusión, otra vez las escuelas públicas surgieron en el discurso de los padres como un lugar al que envir a sus hijos se constituiría como un serio riesgo. De manera similar a lo que Ball (2003) encontró en un grupo de padres de clase media en Inglaterra, los padres de ambas fracciones eligieron escuelas privadas «simple y precisamente porque no son escuelas públicas, porque esto les permite diferenciarse y distanciarse de la escuela pública» (Ball, 2003, p. 56). Varios padres mencionaron que un problema fundamental con las escuelas estatales era precisamente su heterogeneidad, puesto que están abiertas a todos los estudiantes: «en el colegio estatal, va a entrar todo el mundo» (Sr. Falcón, Colegio A). Esto hace referencia a lo que se considera un «tipo inapropiado de heterogeneidad» (Ball, 2003, p. 59), pues «todos» incluye a niños que provienen de familias socialmente en riesgo, con quienes no desean que sus hijos se mezclen, como lo expresaba la Sra. Chura.

De manera similar a lo encontrado en diferentes estudios, lo que los padres buscan evitar son las instituciones educativas con población de estudiantes socialmente mixta (Reay et ál, 2001, Reay y Lucey, 2004; Elacqua, 2012). Con respecto a esto, los comentarios de algunos padres resultaron discriminatorios en relación con las familias que envían a sus hijos a las escuelas estatales, frente a las cuales existe una fuerte estigmatización:

Entrevistadora: ¿Bajo qué condiciones un colegio estatal podría convertirse en una opción atractiva para la educación de sus hijos?

15. Esto resulta consistente con los resultados de diferentes estudios, en los cualse se evidencia que los padres prefieren o eligen escuelas con niños que provienen de la misma condición socioeconómica (Frankenberg et ál., 2010; Saparito, 2003; Buckley y Schneider, 2002 -citados en Elacqua, 2012-). 
Madre: Incluso si todo mejorara en las escuelas públicas y tuvieran buenos profesores, buena infraestructura, buenos materiales... el problema todavía sería las familias que envían a sus hijos allí (Sra. Escobal, Colegio A).

De manera similar a lo encontrado en el estudio de Bagley, Woods y Glatter (2001), los padres entrevistados señalaron que la clase de estudiantes que van a la escuela pública era una de las razones principales para rechazar esta escuela, por la cual la consideraban inapropiada para sus hijos.

Finalmente, se debe destacar que, como cualquier otro habitus, la interiorización de esta sensación de no pertenencia a la escuela pública es inconsciente, y resulta difícil explicarlo porque forma parte de un sentido común. Esto lo expresa una madre del Colegio B, en cuyo proceso de elección de escuela visitó un colegio estatal y refirió lo siguiente: «Yo sé que uno no puede juzgar a los niños y no se trata de eso, pero es difícil de explicar... cuando estuve en esa escuela simplemente no podía imaginar a mi hijo allí. No, no lo veo en un colegio estatal» (Sra. Meza, Colegio B).

El discurso de los padres y madres entrevistados refleja de cerca lo que sentencia Vergara:

la educación pública está reservada únicamente para los más pobres del país, para el concho último de la sociedad [...] Y cada vez que alguien prospera en la escala social, por más nimia que sea su mejora, inmediatamente deserta del colegio público y se traslada a uno privado. Ese colegio particular en los barrios de clase baja (y en muchos de clase media) no es mejor que el estatal; la escuela privada destinada a los ciudadanos de menos recursos no les salva de una educación anémica, solo les ahorra el estigma de haber pasado por un «colegio nacional» (2013, p. 48).

Aquí también resulta relevante la reflexión planteada por Gentilli (2015) en relación con que la molestia de los padres hacia la escuela estatal no representa la diversidad de orígenes de los estudiantes, sino la posiblidad del fracaso: «ir a la escuela pública significa correr el riesgo de ser un perdedor», lo que constituye un referente contrario y opuesto a su trayectoria ascendente, y al posicionamiento actual que desean preservar.

\section{- La búsqueda de estatus y exclusividad como diferenciación social}

Otra forma en la que se expresa la diferenciación social es lo que representa la elección de escuela en términos de búsqueda de estatus y exclusividad a través de dicha elección. Como afirma Ball, «aparte de ventajas materiales que se pueden acumular a través del éxito académico, hay beneficios culturales que se pueden obtener en forma de prestigio social al elegir un determinado tipo de escuela» (1997, p. 2). En una perspectiva similar, Adnett y Davies (2002) se refieren a la educación como bien de consumo, con lo cual aluden a los beneficios 
inmediatos que ofrece la elección de un tipo de colegio por el prestigio social que otorga a las familias. Benavides se refiere a ello cuando afirma que, para los padres en el Perú, «la escuela elegida representa en mayor o menor medida un símbolo de estatus y de realización de su lógica aspiracional» (comunicación personal, 22 de agosto, 2012), que les hace sentir que son miembros de un grupo al que desean pertenecer. Son capaces de permitirse el lujo de los estilos de vida de la clase media, que se manifiesta especialmente en poder comprar una educación privada para sus hijos. En este proceso, se diferencian cada vez más de los miembros de las clases bajas, que no pueden acceder al tipo de educación privada a la que ellos ahora sí tienen acceso.

Dentro de un sistema educativo altamente jerárquico y segregado, el estatus y la exclusividad pueden distinguirse a lo largo del continuum entre las escuelas estatales y las escuelas privadas más exclusivas, pasando por los diferentes circuitos educativos que existen en el sistema y que impiden el contacto de los diferentes sectores sociales. Los padres expresan estas diferentes formas de estatus y exclusividad que otorga la escuela: «Ahora nosotros tenemos cierto estatus en este colegio, comparado con un colegio nacional de repente, de un colegio chiquito» (Sr. Falcón, Colegio B).

Cuando me encuentro con mis compañeras de la universidad también hablamos de la escuela de nuestros hijos, porque siempre es eso ¿no? Y decían: «ay mis hijos están en el Juan XXIII»... Y yo digo: «Mis hijos están en el Colegio A», y normal, ipaso piola! [risas]. Claro, este amigo puso su hija allí [en referencia a una escuela más cara], porque solo tienen una hija y ambos padres trabajan... o una amiga que le fue bien en los negocios y tenía a su hijo en el San Agustín, pero ellos viven en el cerro en Villa María, pero eso les daba caché (Sra. Miranda, Colegio A).

\section{Conclusiones}

El objetivo de este estudio fue contribuir a entender mejor el fenómeno del tránsito de la escuela pública a la escuela privada en el sector emergente en Lima Metropolitana, yendo más allá del argumento de búsqueda de calidad y situándolo también como un fenómeno relacionado a procesos sociales más complejos. Para ello, se analizaron los procesos de elección de escuelas privadas en una muestra comparada de familias emergentes considerando la complementariedad de las perspectivas racionalista y culturalista utilizadas comúnmente en los estudios de elección escolar.

Un primer hallazgo es el valor de la escolaridad como un modo de progreso y movilidad social. Para las madres y padres entrevistados, la escolaridad de sus hijos resulta fundamental para alcanzar sus realizaciones futuras: estudiar en la universidad y convertirse en profesionales. Por ello, como señala la perspectiva racionalista, la elección escolar ha estado basada en un cuidadoso 
cálculo para poder determinar qué alternativa es la que les permitirá asegurar estas realizaciones futuras. En ambos grupos, solo la educación privada cumple con esta expectativa y se decide invertir en ella.

Un segundo hallazgo es la diversidad de criterios que las familias sopesan y someten a evaluación al momento de elegir el colegio. En primer lugar, el costo es lo que diferencia lo posible de lo imposible en la elección escolar, es un es factor que posibilita elegir una marca de privilegio económico. Los otros criterios que se ponen en juego no se refieren únicamente a las características físicas (infraestructura, equipamiento, etc.), sino que dan cuenta de una amplia variedad de expectativas en relación con la escolaridad, que adoptan significados o matices distintos en cada grupo. Lo que se pone en juego al evaluar cada criterio es tanto su aporte a las realizaciones futuras como cuánto se acercan a brindar beneficios inmediatos (sensación de bienestar de los hijos, de seguridad, de atención más personalizada, etc.). La escuela estatal aparece de manera recurrente como alejada o sin posibilidad de cumplir con los criterios esperados por los padres.

Junto con esta aproximación más estratégica, los hallazgos también muestran que los procesos de elección están fuertemente mediados por un conjunto de disposiciones que se van internalizando como sentido común y que han vuelto natural la opción por la escuela privada. Esto se asocia fuertemente con la trayectoria de movilidad ascendente de las familias y con su interés por preservar su actual posicionamiento social. Estas disposiciones dan cuenta de prácticas de identificación social que buscan afianzar las relaciones con «los semejantes», básicamente representados por grupos de padres preocupados y comprometidos con la educación de sus hijos; y, en el caso del grupo más consolidado, por su reconocimiento como gente emprendedora.

Esto tiene su contraparte en las prácticas de diferenciación social, que se vinculan con los esfuerzos por separarse y distinguirse de «los otros», de aquellos grupos con los que no desean ser asociados. Esto dificulta (o imposibilita) concebir la escuela como un espacio de encuentro e inclusión. La mayor estigmatización se dirige hacia la escuela pública. Como plantea Gentilli (2015), la molestia de los padres hacia la escuela estatal está representada en la posiblidad del fracaso: «ir a la escuela pública significa correr el riesgo de ser un perdedor», lo que constituye un referente contrario y opuesto a su trayectoria ascendente y al posicionamiento actual que desean preservar.

Todo lo encontrado en este estudio plantea importantes desafíos al sistema educativo, pues, como viene ocurriendo en otros países, el tránsito de lo público a lo privado contribuye a reforzar y exacerbar la segregación a través de la configuración de «un sistema con circuitos educativos diferenciados que impide el contacto de los diferentes sectores sociales» (Del Cueto 2004). Como ya denunciaban Alberti y Cotler (1977) hace casi cuarenta años, el sistema educativo permite la movilidad social de algunos individuos sin cuestionar la estratificación existente, a lo que agregaría que tiende más bien a normalizarla. Quisiera terminar recalcando que no es mi intención señalar o culpar a actores 
sociales individuales como autores de un sistema injusto que reproduce sistemáticamente la desigualdad. Mi propósito es evidenciar cómo nuestro sistema de elección escolar está exacerbando la desigualdad y la exclusión; y subrayar la necesidad de incluir estas preocupaciones en el debate de lo público y lo privado. Como ya se viene planteando en nuestro vecino país del sur, Chile, urge poder hacer frente de forma simultánea a la segregación y la deficitaria calidad del sistema educativo.

\section{Nota biográfica}

PIlar Sanz es PhD en Educación por la Universidad de Bath, Inglaterra y es máster en Estudios de Desarrollo con especialización en Gestión y Política Pública por el Institute of Social Studies en la Haya, Holanda. Actualmente se desempeña como consultora e investigadora de políticas y programas educativos. Este artículo es parte de su investigación doctoral realizada entre el 2011 y 2015 que se centra en la formación del habitus o disposición por la educación privada en la elección escolar de familias emergentes a través del estudio de las trayectorias educativas familiares.

\section{Referencias}

Adnett, N. y Davies, P. (2002). Education as a Positional Good: Implications for Market-Based Reforms of State Schooling. British Journal of Educational Studies 50(2), 189-205.

Alberti, G. y Cotler, J. (1977). Aspectos sociales de la educación rural en el Perú. Lima: Instituto de Estudios Peruanos.

Ansión, J., Lazarte, A., Matos, S., Rodríguez, J., y Vega-Centeno, P. (1998). Educación: La mejor herencia. Decisiones educativas y expectativas de los padres de familia. Una aproximación empírica. Lima: Fondo Editorial de la Pontificia Universidad Católica del Perú.

Arellano, R. (2010). Al medio hay sitio. El crecimiento social según los estilos de vida. Lima: Planeta.

Bagley, C., Woods, P. A y Glatter, R. (2001). Implementation of school choice policy: Interpretation and response by parents of students with special educational needs. British Educational Research Journal, 27(3), 287-307.

Balarín, M. (2015). Low-fee private schools in Peru. Norrag Newsbite, May 8, 2015. Recuperado de https://norrag.wordpress.com/2015/05/08/low-feeprivate-schools-in-peru/

Ball, S. J., Bowe, R. y Gewirtz, S. (1995). Circuits of schooling: A sociological exploration of parental choice of school in social class contexts. Sociological Review, 43(1), 52-78. 
(1996). School choice, social class and distinction: The realization of social advantage in education. Journal of Education Policy, 11(1), 89-112.

Ball, S. J. (1997). On the cusp: Parents choosing between state and private schools in the UK: Action within an economy of symbolic goods. International Journal Inclusive Education, 1(1), 1-17.

(2003). Class strategies and the educational market. The middle classes and social advantage. Londres: Routledge Falmer

Benavides, M., León, J. y Etesse, M. (2014). Desigualdades educativas y segregación en el sistema educativo peruano. Una mirada comparativa de las pruebas PISA 2000 y 2009. Avances de Investigación. Lima: Grade.

Bourdieu, P. (1986). Distinction: A social critique of the judgement of taste. Londres: Routledge (1990). The logic of practice. Cambridge: Polity Press

Bourdieu, P. y Wacquant, J. D. (1992). An invitation to reflexive sociology. Chicago: University of Chicago Press.

Brown, P. (1990). The 'Third Wave': Education and the ideology of parentocracy. British Journal of Sociology of Education, 11(1), 65-85.

Calónico, S. y Ñopo, H. (2007). Returns to private education in Peru (IZA Discussion Paper 2711). Interamerican Development Bank, Washington D.C.

Cuenca, R. (2013). La escuela pública en Lima Metropolitana. ¿Una institución en extinción? Revista Peruana de Investigación Educativa, (5) 73-98.

Cuenca, R. y Montero, C. (2006). Encuesta Nacional de Educación 2005. Lima: Foro Educativo

Del Cueto, C. (2004). Estrategias educativas de las clases medias en urbanizaciones cerradas del Gran Buenos Aires. Espiral, 9(31), 249-276.

Elacqua, G. (2012). The impact of school choice and public policy on segregation: Evidence from Chile. International Journal of Educacion Development, 32, 444-453

Franco, R., Hopenhayn, M. y Leon, A. (2011). Crece y cambia la clase media en América Latina: Una puesta al día. Revista Cepal, 103.

Gentilli, P. (2015). Escuelas en venta, fraudes, darwinismo pedagógico y otras peculiaridades de la educación privada latinoamericana. Miradas críticas sobre la privatización de (y en) la educación. Recuperado de http://www.campanaderechoeducacion.org/privatizacion/escuelas-enventa-fraudes-darwinismo-pedagogico-y-otras-peculiaridades-de-laeducacion-privada-latinoamericana/

Gewirtz, S., Ball, S. y Bowe, R. (1995). Markets, choice and equity in education. Buckingham: Open University Press. 
Gill, B. (2005). School choice and integration. En J. Betts y T. Loveless (Eds.), Getting choice right. Ensuring equity and efficiency in education policy. Washington D.C.: Brookings Institution Press.

Goldthorpe, J. (1996). Class analysis and the reorientation of class theory: The case of persisting differentials in educational attainment. British Journal of Sociology, 47(3), 481-505.

Hatcher, R. (1998). Class differentiation in education: Rational choices? British Journal of Sociology of Education, 19(1), 5-24.

Hirschman, A. (1970). Exit, Voice and Loyalty. Responses to Decline in Firms, Organizations and State. Harvard University Press.

Jaramillo, F. y Zambrano, O. (2013). La clase media en Perú: Cuantificación y evolución reciente. Lima: Banco Interamericano de Desarrollo.

Kosunen, S. (2013). Reputation and parental logics of action in local school choice space in Finland. Journal of Education Policy, 29(4), 443-466.

Lauder, H., et ál. (1999). Trading in futures. Why markets in education don't work. Buckingham: Open University Press

Montero, C. y Cuenca, R. (2008). Sobre notas y aprendizajes escolares: opiniones y demandas de la población del Perú. Segunda Encuesta Nacional de Educación - Enaed 2007. Lima: Foro Educativo.

Parkin, F. (1979) Marxism and class theory: A bourgeois critique. Londres: Tavistock.

Pedraglio, S. (2003). Los Olivos: Clase a «medias». En J. Gamero y M. Zeballos (Eds.), La clase media ¿existe? Perú Hoy, (4). Lima: Desco.

Reay, D. (1997). Feminist theory, habitus, and social class: Disrupting notions of classlessness. Women's Studies International Forum, 20(2), 225-233.

Reay, D. y Ball S. J. (1998). Making their minds up': Family dynamics of school choice. British Educational Research Journal, 24(4), 431-448.

Reay, D., Davies, J., David, M. y Ball, S. J. (2001). Choices of degree or degrees of choice? Class, «race», and the higher education choice process. Sociology, 35(4), 855-874.

Reay, D. y Lucey, H. (2003). The limits of «choice»: Children and inner city schooling. Sociology, 37(1), 121-142.

. (2004). Stigmatized choices: Social class, social exclusion and secondary school markets in the inner city. Pedagogy, Culture and Society, 12(1), 35-51.

Tiramonti, G. y Ziegler, S. (2008). La educación de las élites. Aspiraciones, estrategias y oportunidades. Buenos Aires: Paidós

Toche, E. (2009). Apuntes sobre las clases medias. Del hortelano su perro. Sin espacio ni tiempo histórico. Perú Hoy, (15). Lima: Desco. 
Unidad de Estadística Educativa - Escale, Ministerio de Educación (s.f.). Magnitudes. Escale. Estadística de la Calidad Educativa. Recuperado de http://escale.minedu.gob.pe/magnitudes

Unidad de Medición de la Calidad Educativa, Ministerio de Educación (2015). Presentación General ECE 2014. Lima: Unidad de Medición de la Calidad Educativa. Recuperado de http://umc.minedu.gob.pe/?p=2338

Vergara, A. (2013). Ciudadanos sin República. ¿Cómo sobrevivir en la jungla política peruana? Lima: Planeta.

Vincent, C. y Ball, S. J. (2006). Children, choice and class practices. Middle-class parents and their children. Londres: Routledge.

$\mathrm{Wu}, \mathrm{X}$. (2011). The power of market mechanism in school choice in three junior middle schools in Nanning: A case study (Tesis de doctorado). University of Bath, Bath. 